\title{
Análisis Rasch del Mini Mental State Examination (MMSE) en adultos mayores de Antioquia, Colombia
}

\author{
Rasch analysis of the Mini Mental State Examination (MMSE) in \\ older adults in Antioquia, Colombia
}
Diego Fernando Rojas-Gualdrón ${ }^{1,2}$ ORCID - Alejandra Segura C. ${ }^{2 \text { SCHOLAR }}$ - Doris Cardona A. SCHOLAR Ángela Segura C. SCHOLAR_María Osley Garzón D. SCHOLAR

'Universidad CES ${ }^{2}$ Fundación Universitaria de San Gil

Colombia

Fecha correspondencia:

Recibido: agosto 17 de 2016. Aceptado: abril 19 de 2017.

Forma de citar:

Rojas-Gualdrón, D.F., Segura C., A., Cardona A., D., Segura C., A., \& Garzón D. M.0. (2017). Análisis Rasch del Mini Mental State Examination (MMSE) en adultos mayores de Antioquia, Colombia. Rev. CES Psico 10(2), 17-27.

\section{Open access}

\section{(c) Copyright}

Licencia creative commons

Ética de publicaciones

Revisión por pares

Gestión por Open Journal System DOl: http://dx.doi.org/10.21615/ cesp.10.2.2

ISSN: 2011-3080

Sobre los autores:

1. Doctorando en Epidemiología y Bioestadística, Magister

Comparte

\section{Resumen}

Objetivo. Evaluar las evidencias de validez del Mini Mental State Examination que sustentan su interpretación en adultos mayores. Métodos. Estudio de fuente secundaria con la participación de 4034 adultos mayores residentes de Antioquia, Colombia, 2012. Se siguieron los lineamientos de Wolfe y Smith para análisis desde el modelo Rasch. Resultados. Se identificó dependencia local entre los ítems de atención y cálculo. Estos producen una segunda medida que afecta la unidimensionalidad del instrumento. La confiabilidad de los ítems fue de 0,80, la medida explicó el 45,2\% de la varianza de las puntuaciones crudas. Conclusión. Los resultados sugieren diferenciar las habilidades de atención y cálculo de los demás procesos cognitivos considerados por el instrumento. El MMSE presenta limitaciones de validez de generalización que potencialmente afectan comparaciones transculturales.

Palabras claves: Salud Mental, Cognición, Anciano, Estudios de Validación

\section{Abstract}

Objective. To evaluate validity of the Mini-Mental State Examination (MMSE) that supports the interpretability in older adults. Methods. Secondary data study administered in 4.034 older adults in Antioquia, Colombia, 2012. Data were analyzed from Rasch models according to Wolfe and Smith guidelines. Results. Attention and calculation items showed local dependency. They produce a second relevant measure affecting the unidimensional type of the test. The reliability of the items was of 0.80 , the measure explained $45.2 \%$ of the raw score variance. Conclusion. Results suggest differentiating attention and calculation abilities from the other cognitive processes considered by the test. MMSE shows generalization validity limitations that may affect transcultural comparisons.

Keywords: Mental Health, Cognition, Aged, Validation Studies 
Pág 18

en Psicología, Magister en E-learning, Psicólogo.

2. Magíster en Epidemiología, Psicóloga.

3. Doctora en Demografía, Magíster en Epidemiología, Magíster en Salud Pública.

4. Doctora en Epidemiología, Magíster en Epidemiología.

5. Magíster en Epidemiología.

\section{Introducción}

La evaluación cognitiva o examen del estado mental de las personas, principalmente en ancianos, puede ser detectado a través de diferentes instrumentos de medición; entre los más utilizados se encuentra el Mini Mental (Mini Mental State Examination - MMSE) construido por Folstein y McHugh en 1975. El Examen del Estado Mental evalúa el comportamiento, orientación, actitud, percepción, juicio, abstracción y cognición del individuo (Bell \& Hall, 1977).

El test es utilizado para detectar deterioro cognitivo y cuantificar su rendimiento global para demencias (Bermejo, Díaz, \& Porta-Etessam, 2001), pero presenta limitaciones cuando se aplica a personas con demencia avanzada, tiene efecto suelo y techo, es sensible a la edad, cultura y educación, y no explora todos los dominios cognitivos (Llamas-Velasco, Llorente-Ayuso, Contador, \& Bermejo-Pareja, 2015), y en las traducciones su comportamiento es diferente (Jones, 2006). Más recientemente se encontró que el MMSE-I (nueva versión) se puede usar en pacientes con demencia (Babacan-Yıldız et al., 2016) y en adultos mayores sanos, para determinar la disminución cognitiva (Kopecek, Bezdicek, Sulc, Lukavsky, \& Stepankova, 2016).

Si bien el MMSE ha sido empleado en diferentes grupos de personas, en la literatura científica se han reportado limitaciones sobre su validez de generalización, aplicabilidad e interpretabilidad. Por ejemplo, Prieto, Delgado, Perea y Ladera (2011) identificaron Funcionamiento Diferencial de los Ítems (DIF) entre pacientes con enfermedad de Parkinson, Alzheimer y sin demencia para 11 de los 30 ítems que componen el instrumento. Esto ha llevado a resaltar otros test cognitivos breves (TCB) de menos costo y más fáciles de aplicar en comparación al MMSE (Carnero-Pardo, 2014). Estos argumentos han sido debatidos por Olazarán y Bermejo (2015).

En el caso particular de Colombia, la escala Mini Mental se ha utilizado como prueba diagnóstica para demencia, según los criterios del DSM-IV. Estudios locales han identificado variaciones según edad y nivel educativo que han llevado a sugerir ajustar las puntuaciones obtenidas en el instrumento en función de estas características para mejorar su desempeño (Rosselli et al., 2000; Pedraza et al., 2014).

A pesar de la amplia disponibilidad de investigaciones sobre las propiedades psicométricas del MMSE a nivel mundial, no se encuentra en la literatura un estudio de validación que retome de forma integral las diferentes facetas de validez reconocidas en la psicometría contemporánea (Wolfe \& Smith, 2007; Messick, 1995); la mayoría de los estudios se han centrado en valorar su confiabilidad y consistencia interna (Olazarán \& Bermejo, 2015). Estos indicadores de desempeño psicométrico no permiten concluir sobre la interpretabilidad de los resultados obtenidos mediante un test limitando su utilidad clínica, máxime dadas las limitaciones de validez de generalización como es el caso del MMSE. Contar con argumentos y evidencias de diferentes facetas de validez, le permite al clínico realizar interpretaciones del estado mental de sus pacientes con base en el conocimiento adquirido sobre su desempeño en el instrumento (Stelmack et al., 2004). De esta forma, en la presente investigación tiene como propósito evaluar las evidencias de validez del Mini Mental State Examination que sustentan la interpretabilidad de sus resultados en adultos mayores, siguiendo los lineamientos de Messick (1995) estructurados por Wolfe y Smith (2007). 


\section{Método}

\section{Diseño}

Estudio de fuente secundaria basado en una investigación transversal sobre la situación de salud de los adultos mayores de Antioquia, Colombia, realizada en 2012 (Arango, Cardona, Duque, Cardona \& Sierra; 2016). La metodología del estudio original ha sido reportada previamente (Cortés-Muñoz, Cardona-Arango, Segura-Cardona, \& Garzón-Duque, 2016).

\section{Participantes}

Un total de 4034 participantes con 60 años de edad o más contestaron el MMSE. Los participantes fueron seleccionados mediante muestreo probabilístico por conglomerados bietápico representativo a nivel de subregiones del departamento de Antioquia. Se excluyeron los residentes de hogares geriátricos puesto que el instrumento fue aplicado en las viviendas a razón del diseño muestral.

\section{Instrumento}

Mini Mental State Examination (MMSE). Creado por Folstein et al. (1975), este instrumento se compone de 30 ítems dicotómicos que evalúan seis procesos cognitivos: Orientación Temporal, Orientación Espacial, Memoria de Fijación, Memoria de Evocación, Atención y Cálculo y Lenguaje.

\section{Procedimiento}

El instrumento fue aplicado por encuestadores en la residencia de cada participante. Los encuestadores recibieron un entrenamiento estandarizado en el protocolo del MMSE, en técnicas de muestreo y en calidad del dato. La información fue complementada con una encuesta de caracterización sociodemográfica y de condiciones de vida del adulto mayor.

\section{Análisis de datos}

Se realizaron los análisis recomendados por Wolfe y Smith (2007) para estudios de validación con el modelo de Rasch de la siguiente forma:

Ajuste y funcionamiento diferencial de los ítems (DIF)

El ajuste de los ítems a los supuestos del modelo de Rasch se valoró mediante medias cuadráticas de Outfit e Infit, correlaciones ítem-medida y análisis de independencia local. Para estos tres estadísticos se tomaron como valores esperados medias cuadráticas ente 0,50 y 2,00, correlaciones ítem-medida mayores a 0,30 y correlaciones entre los residuales de los ítems menores a 0,70 (Bond \& Fox, 2001).

El DIF se valoró para establecer la estabilidad de las dificultades de los ítems entre sexos (hombre-mujer), estatus de enfermedad (enfermo-no enfermo) y edad (menores de 70 años - mayores). Se estimaron los contrastes de DIF y la probabilidad de la prueba de Mantel-Haenzel. Para controlar por falsos positivos debidos a comparaciones múltiples se consideraron como DIF significativos aquellos con valor crítico de Benjamini-Hoschberg menor a la probabilidad de la prueba de Mantel-Haenzel (Thissen, Steinberg, \& Kuang, 2002) y con contraste de DIF mayor a 0,50 logitos y por fuera de los límites de acuerdo de Bland y Altman (1986).

Las dificultades de los ítems del MMSE en los adultos mayores de Antioquia fueron comparadas con las reportadas por Prieto et al. en Castilla y León, España, en 2011 para valorar la estabilidad del MMSE y sus posibilidades de comparación transcultural. 
Unidimensionalidad, Confiabilidad y mapa de Wright

La unidimensionalidad de la medida Rasch derivada del MMSE se determinó mediante dos criterios: a) varianza explicada por la medida mayor a 40\% (Bond, 2004), y b) varianza explicada por el contraste residual más importante menor a 10\%. Se verificó la correlación disatenuada entre la medida Rasch y el primer contraste con valor esperado mayor a 0,50 (Wolfe \& Smith, 2007).

Se calcularon la confiabilidad (Rasch reliability) e índice de separación para los ítems y las personas, al igual que la consistencia interna de las puntuaciones crudas. Se presenta el mapa de Wright y sobre este se analiza el targeting de la escala con respecto a la distribución de las habilidades de los participantes.

Los análisis se realizaron en Winsteps 3.92.1 (Linacre, 2016) y Stata 12 (StataCorp, 2011).

\section{Resultados}

La población estudiada (Tabla 1) correspondió a 4034 personas mayores, la mitad registró una edad de 70 años, 60\% población femenina, residentes principalmente en la zona urbana y predominio de mestizos. Es llamativo que solo un tercio de los adultos mayores reportó haberse enfermado o consultar a los servicios médicos en los últimos cuatro meses de la aplicación de la encuesta.

Tabla 1. Características sociodemográficas de adultos mayores participantes. Antioquia, Colombia 2012

\begin{tabular}{|c|c|c|c|}
\hline Variable & & $N$ & $\%$ \\
\hline Sexo & $\begin{array}{l}\text { Hombre } \\
\text { Mujer }\end{array}$ & $\begin{array}{l}1549 \\
2485 \\
\end{array}$ & $\begin{array}{l}38,40 \\
61,60 \\
\end{array}$ \\
\hline Área de residencia & $\begin{array}{l}\text { Urbana } \\
\text { Rural }\end{array}$ & $\begin{array}{c}3712 \\
322 \\
\end{array}$ & $\begin{array}{c}92,00 \\
8,00 \\
\end{array}$ \\
\hline Etnia & $\begin{array}{l}\text { Mestizo } \\
\text { Otro }\end{array}$ & $\begin{array}{c}3458 \\
576 \\
\end{array}$ & $\begin{array}{l}85,70 \\
14,30 \\
\end{array}$ \\
\hline Enfermedades reportadas en los últimos cuatro meses & $\begin{array}{l}\text { Sí } \\
\text { No }\end{array}$ & $\begin{array}{l}2783 \\
1251 \\
\end{array}$ & $\begin{array}{l}69,00 \\
31,00\end{array}$ \\
\hline Edad & Mediana & \multicolumn{2}{|c|}{$70(64-77)$} \\
\hline
\end{tabular}

\section{Ajuste y funcionamiento diferencial de los ítems (DIF)}

Todos los ítems muestran medias cuadráticas de Outfit en el rango 0,53 - 1,87 y medias cuadráticas Infit en el rango 0,63-1,38. Las correlaciones ítem medida son mayores a 0,30 excepto para los ítems Orientación temporal 2, Orientación espacial 1, y Lenguaje 1. La Tabla 2 muestra los estadísticos de ajuste y las dificultades de los ítems con sus errores estándar expresados en logitos.

Se identificaron problemas de dependencia local ente las tareas de Atención y cálculo con correlaciones entre residuales de los ítems 4 y $5(r=0,95), 3$ y $4(r=0,90) 3$ y $5(r=0,86) .2$ y $3(r=0,74)$ y 1 y $2(r=0,70)$.

La Figura 1 muestra los Límites de acuerdo de Bland y Altman por edad (Figura 1a), sexo (Figura 1b), reporte de enfermedad (Figura 1c) y la comparación de las dificultades de los ítems obtenidas para los adultos mayores de Antioquia con las reportadas 
Tabla 2. Estadísticos de los ítems del MMSE en adultos mayores de Antioquia, Colombia 2012

\begin{tabular}{|c|c|c|c|c|c|}
\hline Ítem & Dificultad & $\begin{array}{c}\text { Error } \\
\text { estándar }\end{array}$ & Outfit & Infit & $\begin{array}{c}\text { Correlación ítem- } \\
\text { medida }\end{array}$ \\
\hline OT1. Orientación temporal 1 & 0,50 & 0,04 & 1,00 & 0,98 & 0,48 \\
\hline OT2. Orientación temporal 2 & $-2,41$ & 0,10 & 1,51 & 1,01 & 0,26 \\
\hline OT3. Orientación temporal 3 & 0,80 & 0,04 & 1,29 & 1,12 & 0,42 \\
\hline OT4. Orientación temporal 4 & $-0,66$ & 0,05 & 0,85 & 0,89 & 0,44 \\
\hline OT5. Orientación temporal 5 & $-0,42$ & 0,05 & 0,80 & 0,89 & 0,46 \\
\hline OE1. Orientación espacial 1 & $-0,88$ & 0,06 & 1,87 & 1,25 & 0,23 \\
\hline OE2. Orientación espacial 2 & 0,17 & 0,04 & 1,22 & 1,09 & 0,40 \\
\hline OE3. Orientación espacial 3 & $-0,24$ & 0,05 & 0,89 & 0,95 & 0,45 \\
\hline OE4. Orientación espacial 4 & $-1,50$ & 0,07 & 1,36 & 1,04 & 0,29 \\
\hline OE5. Orientación espacial 5 & $-2,20$ & 0,09 & 0,84 & 0,93 & 0,32 \\
\hline OE6. Orientación espacial 6 & $-2,16$ & 0,09 & 0,85 & 0,99 & 0,30 \\
\hline MF1. Memoria de fijación 1 & $-2,61$ & 0,10 & 0,64 & 0,90 & 0,31 \\
\hline MF2. Memoria de fijación 2 & $-1,69$ & 0,07 & 1,01 & 0,91 & 0,35 \\
\hline MF3. Memoria de fijación 3 & $-1,39$ & 0,07 & 0,79 & 0,93 & 0,38 \\
\hline AC1. Atención y cálculo 1 & 2,53 & 0,04 & 0,76 & 0,84 & 0,63 \\
\hline AC2. Atención y cálculo 2 & 1,98 & 0,04 & 0,63 & 0,71 & 0,69 \\
\hline AC3. Atención y cálculo 3 & 1,57 & 0,04 & 0,55 & 0,65 & 0,71 \\
\hline AC4. Atención y cálculo 4 & 1,43 & 0,04 & 0,53 & 0,63 & 0,72 \\
\hline AC5. Atención y cálculo 5 & 1,37 & 0,04 & 0,53 & 0,64 & 0,71 \\
\hline ME1. Memoria de evocación 1 & 1,55 & 0,04 & 1,36 & 1,30 & 0,38 \\
\hline ME2. Memoria de evocación 2 & 2,82 & 0,04 & 1,64 & 1,38 & 0,34 \\
\hline ME3. Memoria de evocación 3 & 2,43 & 0,04 & 1,43 & 1,29 & 0,40 \\
\hline LE1. Lenguaje 1 & $-1,68$ & 0,07 & 1,11 & 1,23 & 0,27 \\
\hline LE2. Lenguaje 2 & $-0,65$ & 0,05 & 1,16 & 1,02 & 0,37 \\
\hline LE3. Lenguaje 3 & 0,07 & 0,04 & 1,24 & 1,03 & 0,42 \\
\hline LE4. Lenguaje 4 & $-0,13$ & 0,03 & 0,81 & 1,24 & 0,46 \\
\hline LE5. Lenguaje 5 & 1,39 & 0,04 & 1,30 & 1,14 & 0,45 \\
\hline
\end{tabular}

por Prieto et al., 2011 para adultos mayores de Castilla y León, España. En las figuras los ítems con DIF significativo y relevante son aquellos ubicados por fuera de los Límites de acuerdo de Bland y Altman (verde) y de las líneas de referencia vertical ubicadas en los valores $\pm 0,5$ logitos (negro).

La dificultad de los ítems relacionados con atención y cálculo (AC1-AC5) y con lenguaje (LE1, LE4, LE5) es significativamente mayor para los adultos menores de 70 años; por el contrario, las dificultades de los ítems relacionados con orientación espacial (OT1, OE2, OE3) y temporal (OT3, OT4) son más altas entre los adultos mayores de 70 años que entre los menores. En general, las dificultades de los ítems relacionados con memoria no difieren por grupos de edad. Únicamente la diferencia en OT1 es mayor a 0,5 logitos. Por sexo, se observa una mayor dificultad para los hombres en los ítems relacionados con lenguaje (LE2, LE3) y memoria de evocación (ME1-ME3) y para las mujeres en los ítems relacionados con orientación espacial (OE2-OE4) y atención y cálculo (AC1-AC5). Entre las diferencias por sexo, el contraste es mayor a 0,50 logitos únicamente para los ítems de orientación espacial en contra de las mujeres. Al comparar las dificultades según reporte de enfermedad no se observan diferencias relevantes. 
En relación con la comparación entre las dificultades encontradas en Antioquia y las reportadas en Castilla y León se observan pocos ítems con valores similares. El acuerdo entre medidas se da principalmente en ítems relacionados con tareas de memoria de fijación y orientación espacial y temporal. Para los adultos mayores de Antioquia la mayoría de las tareas relacionadas con el lenguaje implicaron mayor dificultad que para los adultos mayores de Castilla y León. Por el contrario, para los adultos mayores de esta región, la dificultad es mayor en las tareas de atención y cálculo y de orientación espacial en comparación con los adultos mayores de Antioquia.

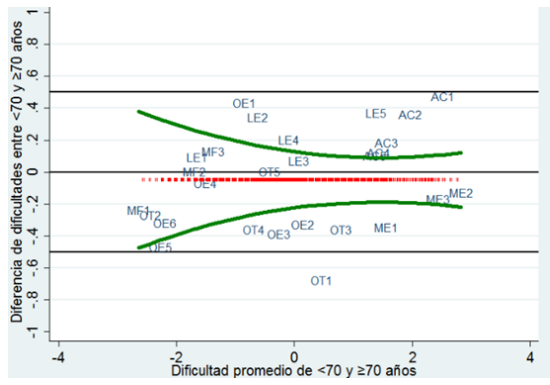

a. Edad (<70 - $\geq 70$ años)

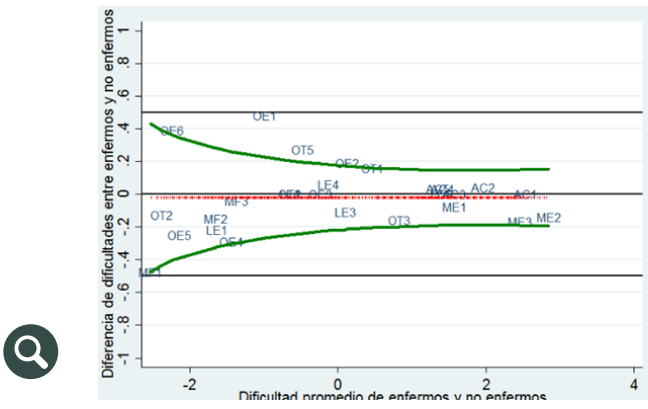

c. Reporte de enfermedad (Sí - No)

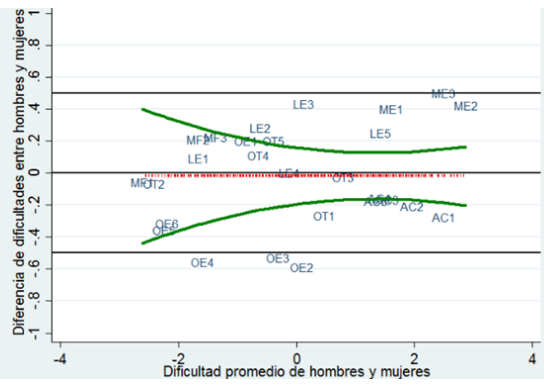

b. Sexo (Hmbre - Mujer)

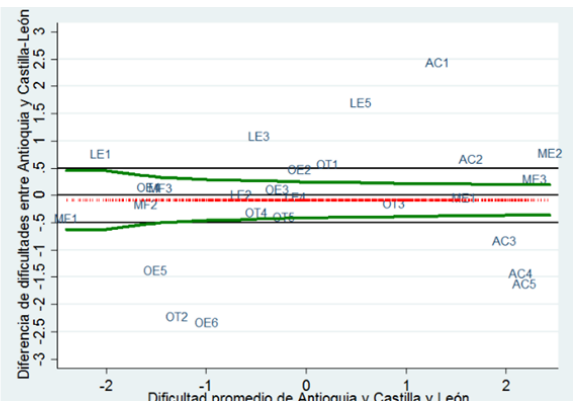

d. Antioquia, 2012 - Castilla y León, 2011

Figura 1. Funcionamiento diferencial de los ítems del MMSE en adultos mayores de Antioquia, Colombia 2012. Líneas verdes: Límites de acuerdo de Bland y Altman, Línea roja: Promedio de las diferencias de dificultades por subgrupo, todos los ítems. Las diferencias (eje vertical) y promedios (eje horizontal) de dificultades de los ítems están expresados en logitos. Los nombres cortos de los ítems se corresponden con los presentados en la Tabla 2.

\section{Unidimensionalidad, confiabilidad y mapa de Wright}

Se obtuvo un valor de 0,80 para la confiabilidad tanto de los ítems como de las personas, lo cual implica un índice de separación de 2. La consistencia interna de las puntuaciones brutas fue de 0,85. La medida Rasch construida a partir del MMSE explicó el 45,20\% de la varianza de las puntuaciones crudas. A partir del análisis de componentes principales realizado sobre los residuales de esta medida se encontró que el primer contraste explicó el 9,60\% de la varianza residual y mostró una correlación disatenuada de 0,32 con respecto a la medida principal. Al revisar la composición de este contraste, se identificó que su primer clúster con cargas factoriales positivas y mayores a 0,50 estaba compuesto por los ítems de atención y cálculo.

La Figura 2 muestra el mapa de Wright para el MMSE. Se observa que el nivel promedio de habilidad de los adultos mayores (M izquierda) es mayor que el valor promedio más una desviación estándar de las dificultades de los ítems de la escala (S derecha). Este desfase entre distribuciones implica a su vez que las medidas de 
atributo mayores a aproximadamente 3 logitos ( $\geq 27$ en puntuación cruda) no cuentan con ítems de dificultad suficiente para ser caracterizadas. El 4,2\% (168) de los adultos mayores obtuvo la puntuación máxima posible y el 0,3\% (11) la puntuación mínima posible. En el mapa también se observa que los ítems que implican mayor dificultad son los relacionados con memoria de evocación y con atención y cálculo con dificultades mayores a 1,00 logito.

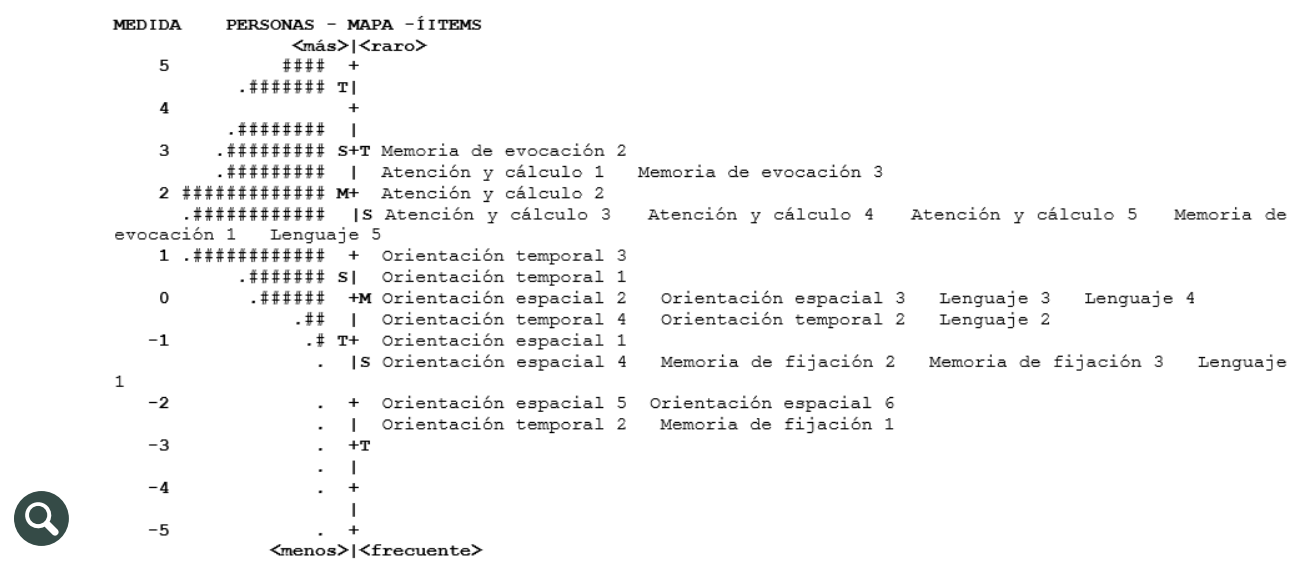

Figura 2. Mapa de Wright del MMSE en adultos mayores de Antioquia, Colombia 2012. M: Promedio, S: una desviación estándar, T: Dos desviaciones estándar. Las medias se presentan en logitos.

\section{Discusión}

El propósito de esta investigación fue evaluar las evidencias de validez del Mini Mental State Examination siguiendo la metodología de Wolfe y Smith (2007) para análisis desde el modelo de Rasch. A continuación, se presentan las interpretaciones de los resultados por facetas de validez según propuesta de Messick (1995).

Validez sustantiva. El rango de puntuaciones del MMSE fue suficiente dado que en conjunto menos del $5 \%$ de los adultos mayores obtuvo las puntaciones extremas de 0 y 30 puntos. La jerarquía de los ítems reflejada en el mapa de Wright se comporta conforme a lo esperado según otros estudios que han identificado como las tareas relacionadas con procesos de atención y memoria conllevan mayor dificultad que tareas que involucran otros procesos cognitivos (Fliss, Lemerre, \& Mollard, 2016; Requena, Turrero, \& Ortiz, 2016). No obstante, el rango de dificultad de los ítems fue insuficiente para describir apropiadamente el nivel de habilidad de los adultos mayores con puntuación cruda $\geq 27$. Si bien el MMSE suele interpretarse con base en puntos de corte menores a este valor, se requiere identificar nuevos ítems con dificultad mayor a los ítems que actualmente conforman el instrumento para poder caracterizar apropiadamente el estado cognitivo de los adultos mayores que logran mayor desempeño en el MMSE.

Validez de contenido. Si bien, como se comentó en el párrafo anterior, la posición en el mapa de Wright de los ítems relacionados con atención y cálculo es acorde a la jerarquía de los ítems, estos mostraron dependencia local entre sí violando uno de los supuestos más relevantes del modelo de Rasch (Engelhard, 2013). Una alternativa viable para evitar este problema sería buscar un sistema de calificación conjunta a partir de los cinco ítems. Las medias cuadráticas Outfit e Infit obtenidas sugieren ajuste apropiado de los ítems al modelo. Su interpretación debe hacerse con precaución a razón de la dependencia local. 
Pág 24

La confiabilidad y consistencia interna fueron moderadas permitiendo separar la información de personas e ítems en dos estratos. Se identificaron varios ítems con DIF entre subgrupos de edad con corte a 70 años y entre sexos, los cuales fueron estadísticamente significativos, pero de poca relevancia clínica. No obstante, dado que los ítems que difieren en dificultad entre estos dos grupos se separan con cierta claridad según el proceso psicológico involucrado en la tarea, es necesario revisar la posibilidad de generalización del instrumento desde su definición operacional.
Validez estructural. La medida logró explicar más del 40\% de la varianza de las puntuaciones brutas, criterio planteado en la literatura como suficiente (Bond \& Fox, 2001). No obstante, el primer contraste entre los residuales, el cual se conforma de los ítems de Atención y cálculo, mostró muy baja correlación disatenuada con la medida principal consolidándose como una segunda medida independiente y relevante obtenida a partir del MMSE en adultos mayores. En este punto es importante aclarar que las medidas construidas desde el modelo de Rasch son unidimensionales por propiedades de la medición conjunta, la segunda medida que se identificó surge de los residuales de la medida principal (Engelhard, 2013). Sin embargo, dadas las condiciones de dependencia local de los ítems de Atención y Cálculo es difícil determinar la validez de generar una segunda medida a partir del MMSE relacionada con estos procesos, la cual sea complementaria a la medida de funcionamiento general que originalmente propone el instrumento. Teóricamente, dada la importancia de las funciones de memoria en la evaluación del adulto mayor, ésta pudiese ser una alternativa válida y útil (Creavin et al., 2016). Se sugiere explorar esta situación con mayor detalle en futuras investigaciones.

Validez de generalización. La confiabilidad y consistencia interna fueron moderadas permitiendo separar la información de personas e ítems en dos estratos. Se identificaron varios ítems con DIF entre subgrupos de edad con corte a 70 años y entre sexos, los cuales fueron estadísticamente significativos, pero de poca relevancia clínica. No obstante, dado que los ítems que difieren en dificultad entre estos dos grupos se separan con cierta claridad según el proceso psicológico involucrado en la tarea, es necesario revisar la posibilidad de generalización del instrumento desde su definición operacional. Por otra parte, de la comparación de las dificultades del MMSE en Antioquia con las reportadas en Castilla y León se concluye que el MMSE parece ser un instrumento limitado para los propósitos de las comparaciones transculturales. Como lo planteó Carnero-Pardo en 2014, posiblemente pueda identificarse una forma corta que retome los ítems con mejores evidencias de validez que produzcan resultados más robustos y comparables entre países y otros grupos relevantes. En este sentido resaltan algunos esfuerzos en Europa para mejorar la comparabilidad de este instrumento (Tennant et al., 2004).

La interpretación de los resultados debe contextualizarse en función de las limitaciones y fortalezas de la investigación. La etnia y el estado de salud fueron establecidos por auto-reporte de los adultos mayores, lo cual podría generar sesgos de información. No obstante esta limitación, los resultados obtenidos sobre funcionamiento diferencial por estas dos características son coherentes con la literatura.

Por otra parte, este trabajo tiene dos fortalezas principales. En primer lugar, se realizó desde el marco epistemológico y metodológico de la medición invariante, operacionalizado en el modelo de Rasch (Engelhard, 2013). En este sentido, es importante resaltar que, si bien cada vez son más frecuentes las publicaciones que recurren a los modelos de Rasch o de Teoría de la Respuesta al Ítem, son pocas las investigaciones que se realizan desde el enfoque contemporáneo de la validez. La fuerza de la psicometría contemporánea no radica exclusivamente en la sofisticación de sus técnicas, sino que se soporta principalmente en sus avances teóricos y epistemológicos en torno a la lógica de la validez y el concepto de medición (Kane, 2001). En segundo lugar, los análisis se realizaron sobre una muestra de gran tamaño, la cual fue obtenida de forma probabilística. Si bien por propiedades de objetividad específica los estadísticos de los ítems son independientes de la muestra (Fischer \& Molenaar. 1995), haber recurrido a un muestreo probabilístico hace que el mapa de Wright 
sea informativo tanto por las locaciones de los ítems, como por la distribución de puntuaciones de los adultos mayores. Esto habilita los resultados derivados de esta investigación para usarse en interpretaciones normativas o basadas en criterio, mejorando la interpretabilidad clínica de los resultados obtenidos por un individuo en el MMSE (Stelmack et al., 2004).

En conclusión, es importante revisar los argumentos y evidencias en pro y en contra de sintetizar las capacidades cognitivas del adulto mayor en una única medida de desempeño global a la luz de los desarrollos recientes. Esto implica una revisión de la definición conceptual y operacional que sustenta el MMSE, principalmente por las limitaciones a su generalización transcultural y por subgrupos como las identificadas en esta investigación y en otros trabajos disponibles en la literatura. No obstante, el MMSE muestra argumentos suficientes para su uso con adultos mayores siempre y cuando se reconozcan sus limitaciones.

\section{Referencias}

Babacan-Yıldız, G., Ur-Özçelik, E., Kolukısa, M., Ișık, A. T., Gürsoy, E., Kocaman, G., \& Celebi, A. (2016). [Validity and Reliability Studies of Modified Mini Mental State Examination (MMSE-E) For Turkish Illiterate Patients With Diagnosis of Alzheimer Disease]. Türk Psikiyatri Dergisi = Turkish Journal of Psychiatry, 27(1), 41-46.

Bell R, \& Hall RC. (1977). The mental status examination. American Family Physician, 16(5), 635-641. Recuperado a partir de http://search.ebscohost.com/login.aspx?direct $=$ true \& $d b=m d c \& A N=920569 \&$ site $=$ eds-live

Bermejo Pareja, F., Díaz Guzmán, J., \& Porta-Etessam, J. (2001). Cien escalas de interés en neurología clínica. Barcelona: Prous Science.

Bland, J. M., \& Altman, D. G. (1986). Statistical methods for assessing agreement between two methods of clinical measurement. Lancet, 1(8476), 307-310.

Bond, T. (2004). Validity and assessment: a Rasch measurement perspective. Metodología de las Ciencias del Comportamiento, 5, 179-194.

Bond, T. G., \& Fox, C. M. (2001). Applying the Rasch Model: Fundamental Measurement in the Human Sciences. Mahwah, NJ: Erlbaum.

Arango, D. C., Cardona, A. S., Duque, M. G., Cardona, A. S., \& Sierra, S. M. C. (2016). Health status of elderly persons of Antioquia, Colombia. Revista Brasileira de Geriatria E Gerontologia, 19(1), 71-86. https://doi.org/10.1590/1809-9823.2016.14199

Carnero-Pardo, C. (2014). ¿Es hora de jubilar al Mini-Mental? Neurología, 29(8), 473481. http://doi.org/10.1016/j.nrl.2013.07.003

Cortés-Muñoz, C., Cardona-Arango, D., Segura-Cardona, Á., \& Garzón-Duque, M. O. (2016). Demographic, social, physical and mental factors associated with functional capacity in the elderly, Antioquia, Colombia, 2012. Revista de Salud Pública, 18(2), 167-178. http://doi.org/10.15446/rsap.v18n2.49237

Creavin, S. T., Cullum, S. J., Haworth, J., Wye, L., Bayer, A., Fish, M., ... Ben-Shlomo, Y. (2016). Towards improving diagnosis of memory loss in general practice: TIMeLi diagnostic test accuracy study protocol. BMC Family Practice, 17(1), 79. http://doi. org/10.1186/s12875-016-0475-2

Engelhard, G. (2013). Invariant measurement: using Rasch models in the social, behavioral, and health sciences. New York: Routledge.

Fischer, G. H., \& Molenaar, I. W. (1995). Rasch Models: Foundations, Recent Developments, and Applications. New York: Springer.

Fliss, R., Lemerre, M., \& Mollard, A. (2016). Performances on a cognitive theory of mind task: specific decline or general cognitive deficits? Evidence from normal aging. Geriatrie Et Psychologie Neuropsychiatrie Du Vieillissement, 14(2), 221-230. http://doi.org/10.1684/pnv.2016.0603 
Folstein, MF., Folstein, SE., \& McHugh, PR. (1975). «Mini-mental state». A practical method for grading the cognitive state of patients for the clinician. Journal of Psychiatric Research, 12(3), 189-198. Recuperado a partir de http://search.eb-

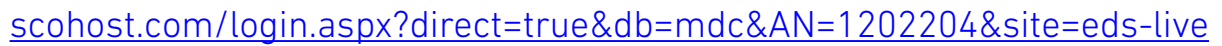

Jones, R. N. (2006). Identification of measurement differences between English and Spanish language versions of the Mini-Mental State Examination. Detecting differential item functioning using MIMIC modeling. Medical Care, 44(11 Suppl 3), S124-133. http://doi.org/10.1097/01.mlr.0000245250.50114.0f

Kane, M. T. (2001). Current Concerns in Validity Theory. Journal of Educational Measurement, 38(4), 319-342.

Kopecek, M., Bezdicek, O., Sulc, Z., Lukavsky, J., \& Stepankova, H. (2016). Montreal Cognitive Assessment and Mini-Mental State Examination reliable change indices in healthy older adults. International Journal of Geriatric Psychiatry. http:// doi.org/10.1002/gps.4539

Linacre, J. M. (2016). Winsteps ${ }^{\circledR}$ Rasch measurement computer program. (Versión 3.92.1). Beaverton, Oregon. Recuperado a partir de www.winsteps.com

Llamas-Velasco, S., Llorente-Ayuso, L., Contador, I., \& Bermejo-Pareja, F. (2015). [Spanish versions of the Minimental State Examination (MMSE). Questions for their use in clinical practice]. Revista De Neurologia, 61(8). Recuperado a partir de http://

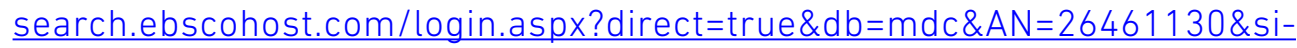
te=eds-live

Messick, S. (1995). Validity of psychological assessment: Validation of inferences from persons' responses and performances as scientific inquiry into score meaning. American Psychologist, 50(9), 741-749. http://doi.org/10.1037/0003$\underline{066 \times .50 .9 .741}$

Olazarán Rodríguez, J., \& Bermejo Pareja, F. (2015). No hay razones científicas para jubilar al MMSE. Neurología, 30(9), 589-591. http://doi.org/10.1016/i. nrl.2014.03.011

Pedraza, O., Sánchez, E., Plata, S. J., Montalvo, C., Galvis, P., Chiquillo, A., \& Arévalo-Rodríguez, I. (2014). Puntuaciones del MoCA y el MMSE en pacientes con deterioro cognitivo leve y demencia en una clínica de memoria en Bogotá. Acta Neurológica Colombiana, 30(1), 22-31.

Prieto, G., Delgado, A. R., Perea, M. V., \& Ladera, V. (2011). Funcionamiento diferencial de los ítems del test Mini-mental en función de la patología. Neurología, 26(8), 474-480. http://doi.org/10.1016/j.nrl.2011.01.013

Requena, C., Turrero, A., \& Ortiz, T. (2016). Six-Year Training Improves Everyday Memory in Healthy Older People. Randomized Controlled Trial. Frontiers in Aging Neuroscience, 8, 135. http://doi.org/10.3389/fnagi.2016.00135

Rosselli, D., Ardila, A., Pradilla, G., Morillo, L., Bautista, L., Rey, O., \& Camacho, M. (2000). [The Mini-Mental State Examination as a selected diagnostic test for dementia: a Colombian population study. GENECO]. Revista De Neurologia, 30(5), 428-432.

StataCorp. (2011). Stata Statistical Software (Versión 14). College Station, TX: StataCorp LP.

Stelmack, J., Szlyk, J. P., Stelmack, T., Babcock-Parziale, J., Demers-Turco, P., Williams, R. T., \& Massof, R. W. (2004). Use of Rasch person-item map in exploratory data analysis: A clinical perspective. Journal of Rehabilitation Research \& Development, 41(2), 233-241.

Tennant, A., Penta, M., Tesio, L., Grimby, G., Thonnard, J.-L., Slade, A., ... Phillips, S. (2004). Assessing and adjusting for cross-cultural validity of impairment and activity limitation scales through differential item functioning within the framework of the Rasch model: the PRO-ESOR project. Medical Care, 42(1 Suppl), 13748. http://doi.org/10.1097/01.mlr.0000103529.63132.77 
Thissen, D., Steinberg, L., \& Kuang, D. (2002). Quick and Easy Implementation of the Benjamini-Hochberg Procedure for Controlling the False Positive Rate in Multiple Comparisons. Journal of Educational and Behavioral Statistics, 27(1), 77-83. http://doi.org/10.3102/10769986027001077

Wolfe, E. W., \& Smith, E. V., Jr. (2007). Instrument development tools and activities for measure validation using Rasch models: part Il--validation activities. Journal of Applied Measurement, 8(2), 204-234. 